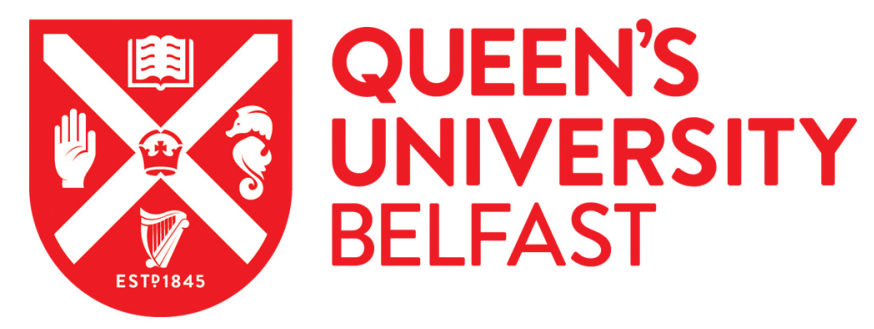

\title{
Robust Fault Tolerant Control of Robot Manipulators with Global Fixed-Time Convergence
}

Van, M., \& Ceglarek, D. (2020). Robust Fault Tolerant Control of Robot Manipulators with Global Fixed-Time Convergence. Journal of the Franklin Institute. https://doi.org/10.1016/j.jfranklin.2020.11.002

Published in:

Journal of the Franklin Institute

Document Version:

Peer reviewed version

Queen's University Belfast - Research Portal:

Link to publication record in Queen's University Belfast Research Portal

Publisher rights

(c) 2020 Elsevier

This manuscript is distributed under a Creative Commons Attribution-NonCommercial-NoDerivs License

(https://creativecommons.org/licenses/by-nc-nd/4.0/), which permits distribution and reproduction for non-commercial purposes, provided the author and source are cited..

\section{General rights}

Copyright for the publications made accessible via the Queen's University Belfast Research Portal is retained by the author(s) and / or other copyright owners and it is a condition of accessing these publications that users recognise and abide by the legal requirements associated with these rights.

Take down policy

The Research Portal is Queen's institutional repository that provides access to Queen's research output. Every effort has been made to ensure that content in the Research Portal does not infringe any person's rights, or applicable UK laws. If you discover content in the Research Portal that you believe breaches copyright or violates any law, please contact openaccess@qub.ac.uk. 


\title{
Robust Fault Tolerant Control of Robot Manipulators with Global Fixed-Time Convergence
}

\author{
Mien $\operatorname{Van}^{1}$ \\ School of Electronics, Electrical Engineering and Computer Science, Queen's University Belfast, Belfast, \\ United Kingdom \\ Dariusz Ceglarek \\ Warwick Manufacturing Group, University of Warwick, Coventry, United Kingdom (e-mail: \\ D.J.Ceglarek@warwick.ac.uk)
}

\begin{abstract}
In this paper, a robust fault tolerant control, which provides a global fixed-time stability, is proposed for robot manipulators. This approach is constructed based on an integration between a fixed-time second-order sliding mode observer (FxTSOSMO) and a fixed-time sliding mode control (FxTSMC) design strategy. First, the FxTSOSMO is developed to estimate the lumped disturbance with a fixed-time convergence. Then, based on the obtained disturbance estimation, the FXTSMC is developed based on a fixed-time sliding surface and a fixed-time reaching strategy to form a global fixedtime convergence of the system. The proposed approach is then applied for fault tolerant control of a PUMA560 robot and compared with other state-of-the-art controllers. The simulation results verify the outstanding fault estimation and fault accommodation capability of the proposed fault diagnosis observer and fault tolerant strategy, respectively.
\end{abstract}

Keywords: Control of robots; fixed-time convergence; sliding mode control; fault tolerant control; robot manipulators.

${ }^{1}$ Email: Corresponding author (m.van@qub.ac.uk)

Preprint submitted to Journal of the Franklin Institute

September 7, 2020 


\section{Introduction}

In order to enhance the quality and quantity of the products in manufacturing, robot manipulators have been widely applied in many sectors [1], [2]. Recently, it is highly demanded that robot and human can work collaboratively to strengthen the advantages

5 of both in manufacturing environment, and a new concept named 'cobot' has been initialized [3]. One of the most important issues in human-robot collaboration is of safety issue [4], [5]. To enhance safety and reliability of robots, fault diagnosis (FD) and fault tolerant control (FTC) have been extensively investigated recently [6], [7], [8]. The goal of the FD is to detect and identify the presence of faults, while the goal 10 of the FTC is to accommodate the effects of faults so that the system can still obtain the desirable tracking performance despite the existing of the faults.

Fault diagnosis (FD) and Fault Tolerant Control (FTC) of robot manufacturing cell which include robot manipulators are significant enablers of Industry 4.0 and can provide an important framework integrating facilitators such as big data, in-line/inprocess monitoring, robotics and control/AI algorithms towards achieving near-zerodefect manufacturing and providing human-robot collaboration capability [9]. There is an increasing number of applications which can significantly benefit from having advanced FD/FTC approaches to increase robot availability and reduce the risk of quality problem and safety hazards in manufacturing. Often these applications require robotic trajectory planning and control under various local faults which can be prevented from developing into product quality/six-sigma failures through the application of advanced FD/FTC approaches. For example, FD/FTC approaches can help in case of (i) robotic laser welding process guided by real-time seam tracking (welding on the fly) as the weld quality depends on integrated control of robotic trajectory planning, laser power and beam modulation parameters [10], [11]; (ii) robotic-inspection with 3D optical/laser scanners placed as in-line measurements conducted within the flowthrough of the production line as the measurement coverage, accuracy, repeatability and cycle time depends on control of robotic path planning [12], [13]; or, (iii) robotic handling of deformable sheet metal parts in multi-press stamping line [14] as the cycle time depends on optimization and control of robotic trajectory planning. One of the 
key requirements of the aforementioned applications is that stability and convergence of the system need to be guaranteed within a fixed and predefined time which often depends on production cycle time and tasks which need to be conducted within the cycle time. Additionally, the stability and convergence time should not depend on the initial state values and it can be computed in advance. However, the current FD/FTC approaches encounter significant limitations as discussed below.

Generally, FTC system can be performed by using either active or passive approach [15]. In the passive approach, i.e., passive fault tolerant control (PFTC), a robust controller is used for both normal and fault operations without requiring an online fault information feedback [16], [17]. The major merit of the PFTC is that it is simpler in design and can compensate the effects of faults quicker. However, it requires a prior knowledge of the bounded value of the fault function. In contrast, in the active approach, i.e., active fault tolerant control (AFTC), the nominal controller of the system is reconfigured based on the fault information, which is obtained based on a fault diagnosis observer [18], [19]. Based on the principle operation of the AFTC, its performance surpasses the PFTC when the fault diagnosis observer provides high precision of fault information. Therefore, several fault diagnosis observers have been developed to accurately estimate fault information, including linear observer [20], high gain observer (HGO) [21], neural network observer (NNO) [22], fuzzy logic observer [23, 24] or slid50 ing mode observer (SMO) [25], etc. Among them, SMO usually provides higher estimate accuracy due to its higher robustness property. Unfortunately, the conventional SMO provides chattering and therefore, it usually requires a low-pass filter to extract the useful information of fault [25]. Nevertheless, the use of the filtration process would destroy the shape of the fault estimation if the filter's parameters are not appropriately selected. Consequently, the system provides lower accuracy of the fault estimation. In order to eliminate the chattering, higher-order sliding mode observer (HOSMO) has been developed for the design of fault diagnosis observer [26]. Unfortunately, the conventional HOSMO does not provide fixed-time convergence. Futhermore, according to the author's knowledge, there have been no fault diagnosis observers in the literature that provide fixed-time convergence for robot manipulators. This motivates us to develop a fault diagnosis observer which can reconstruct the fault information without 
using any filters and guarantee a fixed-time convergence.

After a fault is detected by a FD observer, it is expected that the controller of the system can be reconfigured to handle the unexpected motions of the robot due to the effects of the fault so as to maintain the desired performance of the system. For robotic systems, several control methododologies have been extensively developed to enhance tracking performance. For example, in [27] and [28], the modified computed torque controllers (CTCs) have been developed. In [29], a backstepping neural network has been developed for the design of FTC system. In [30], an adaptive controller based on fuzzy neural network (FNN) has been developed. In [31], a FTC is designed based on the measurement of position only. A new FTC, which is an integration between an adaptive control reconfiguration approach and a control reallocation mechanism, has been proposed in [32]. Recently, conventional sliding mode control (SMC) has been employed to the design of FTC systems due to its high robustness against the system uncertainties and disturbances [33], [34], [35], [36]. In [37], a new FTC scheme based on an integral sliding mode controller has been developed. However, the conventional SMC does not provide finite time convergence. In order to obtain finite time stability characteristic, terminal sliding mode controllers (TSMCs) have been developed [38], [39]. Unlike the conventional SMC, which uses a linear sliding surface in the design, the TSMC uses a nonlinear sliding surface so that the system can obtain a finite time convergence. This merit feature of the TSMC has been employed in the design of FTC for robot manipulators in our previous works [40] and [41]. As the results reported in [40] and [41], the uses of the TSMCs for the FTC system preserve many outstanding features than that of the conventional SMC, such as higher tracking precision, faster transient response and faster convergence. However, the convergence speed of the conventional TSMC depends on the initial state values, which provides a longer convergence time when the initial state values are big. In order to circumvent this shortcoming, a new advanced SMC, namely fixed-time sliding mode control (FxTSMC), has been proposed recently [42, 43, 44]. The advantage of the FxTSMC is 90 that the stability and convergence of the system can be guaranteed within a fixed-time, which does not depend on the inital state values and can be computed in advance. This property is motivated by many practical applications, which requires a faster conver- 
gence and the operators need to know the bound of the convergence time in advance. Unfortunately, there have been no approaches in the literature to design a fixed-time convergence controller for the FTC system of robot manipulators, which motivates us to develop a new FTC scheme, which can guarantee a fixed-time convegence for robot systems.

In summary, two motivations inspire this work: (i) it is neccessary to develop a fault diagnosis observer, which can guarantee the observation error converged within a fixed-time, to estimate the fault information directly without the use of any filters; (ii) it is neccessary to develop a FTC system, which can provide a fixed-time convergence. In addition, it is desirable that the closed-loop observer-controller error signals can be convergent within a fixed-time. In this paper, a new FTC scheme, which guarantees a global fixed-time convergence, is proposed for robot manipulators. The proposed scheme includes two new fixed-time sliding mode schemes. In the first scheme, a fixedtime super-twisting sliding mode observer is developed to obtain an exact fault information after a fixed time, where the convergence time can be calculated and bounded by a constant. The estimated fault information can be utilized to detect and isolate the fault location. Then, a fixed-time FTC is designed based on the obtained fault information to compensate the effects of faults in the system. The fixed-time stability and convergence of the closed-loop system is guaranteed and can be computed in advance. Finally, the proposed fault diagnosis observer and FTC are applied for a PUMA560 robot and compared with other state-of-the-art methods. The comparison results demonstrate the superiority performance of the proposed method.

In summary, the main contributions and novelties of this paper can be highlighted as follows:

- Compared to the existing fault diagnosis observers such as linear observer [20], high gain observer (HGO) [21], neural network observer (NNO) [22] or sliding mode observer (SMO) [25], a new fixed-time super-twisting sliding mode observer is propropsed. The proposed observer provides many outstanding features, such as higher estimation precision is obtained and the estimation error of the proposed observer is converged within a fixed time, which can be computed 
in advance.

- Compared to the existing FTCs, e.g. [40] and [41], for robot manipulators, a new fixed time FTC based on a nonsingular fixed-time sliding mode control, which can guarantee a fixed-time convergence of the tracking errors, is proposed.

- The stability and convergence of the closed-loop system are guaranteed after a fixed time, which is unprecedented for the FTC systems of robot manipulators.

The remaining of the paper is organized as follows. In section 2, problem statement and some definitions about finite-time stability and fixed-time stability are introduced. The fixed-time sliding mode observer and the fixed-time second-order sliding mode observer for fault diagnosis are presented in section 3. In section 4, the design of FTC based on a fixed-time sliding mode controller is described. The simulation results are shown to demonstrate the effectiveness of the proposed algorithm, in section 5. Section 6 provides conclusions.

\section{Problem Statement and Definitions}

\subsection{Problem statement}

Without loss of generality, the following robot dynamics is considered:

$$
\ddot{q}=M^{-1}(q)\left(\tau-C(q, \dot{q}) \dot{q}-F(\dot{q})-G(q)-\tau_{d}\right)+\gamma\left(t-T_{f}\right) \phi(q, \dot{q}, \tau)
$$

where $q \in \mathfrak{R}^{n}, \dot{q} \in \mathfrak{R}^{n}$ and $\ddot{q} \in \mathfrak{R}^{n}$ represents the position, velocity and acceleration of the robot, respectively. $\tau \in \mathfrak{R}^{n}$ is the actuation inputs. The inertia matrix $M(q) \in \mathfrak{R}^{n \times n}$ is positive and definite. $C(q, \dot{q}) \in \mathfrak{R}^{n}$ consists of the Coriolis and Centripetal forces. $F(\dot{q}) \in \mathfrak{R}^{n}$ is the friction matrix. $\tau_{d}$ stands for load disturbance matrix and $G(q) \in \mathfrak{R}^{n}$ indicates the vector of gravity terms. The vector $\phi(q, \dot{q}, \tau) \in \mathfrak{R}^{n}$ represents the possible fault components in the system. The vector $\gamma\left(t-T_{f}\right)$ denotes the time profile of the faults, in which $T_{f}$ is the time of occurrence of the faults.

The robot dynamics considered in (1) is assumed to satisfy the following standard property :

$$
0<\lambda_{m}\{M(q)\} \leq\|M(q)\| \leq \lambda_{M}\{M(q)\} \leq \Upsilon, \Upsilon>0
$$


In the above equation, $\lambda_{m}\{M\}$ and $\lambda_{M}\{M\}$ indicates the minimum and maximum eigenvalues of matrix $M$, respectively.

The matrix $\gamma(\cdot)$ is configured as

$$
\gamma\left(t-T_{f}\right)=\operatorname{diag}\left\{\gamma_{1}\left(t-T_{f}\right), \gamma_{2}\left(t-T_{f}\right), \ldots, \gamma_{n}\left(t-T_{f}\right)\right\}
$$

where $\gamma_{i}$ represents the fault component existing in the $i$ th state equation.

The time profile of each state equation is introduced by [41]:

$$
\gamma_{i}\left(t-T_{f}\right)= \begin{cases}0, & \text { if } t<T_{f} \\ 1-e^{-l_{i}}\left(t-T_{f}\right), & \text { if } t \geq T_{f}\end{cases}
$$

where $i_{i}>0$ indicates the development of the fault. When the value of $t_{i}$ is small, incipient faults are presumed. In contrast, when the value of $t_{i}$ is large, the time profile $\gamma_{i}$ represents abrupt faults.

The model (1) can be rearranged as follows:

$$
\begin{aligned}
\ddot{q}= & M^{-1}(q) \tau+M^{-1}(q)(-C(q, \dot{q}) \dot{q}-G(q)) \\
& +M^{-1}(q)\left(-F(\dot{q})-\tau_{d}\right)+\gamma\left(t-T_{f}\right) \phi(q, \dot{q}, \tau)
\end{aligned}
$$

The following state space model can be obtained from (5) by introducing $x_{1}=q$ and $x_{2}=\dot{q}$ :

$$
\begin{aligned}
& \dot{x}_{1}=x_{2} \\
& \dot{x}_{2}=\Lambda u+f\left(x_{1}, x_{2}\right)+\delta\left(x_{1}, x_{2}, u\right)+\Xi
\end{aligned}
$$

where $\Lambda=M^{-1}(q), f\left(x_{1}, x_{2}\right)=M^{-1}(q)(-C(q, \dot{q}) \dot{q}-G(q))$ denotes the lumped known component, $\delta\left(x_{1}, x_{2}, u\right)=M^{-1}(q)(-F(\dot{q}))+\gamma\left(t-T_{f}\right) \phi(q, \dot{q}, \tau)$ denotes the lumped uncertainty and $\Xi=M^{-1}(q) \tau_{d}$ denotes the lumped disturbance component in the system.

The objective of this paper is to design a FTC input $u$ such that the system can tolerate the effects of the uncertainties, disturbances and faults well and guarantee that the desirable tracking performance of the system can be achieved.

Assumption 1. The unknown function is bounded by

$$
\left|\Omega\left(x_{1}, x_{2}, u, t\right)\right|=\left|\delta\left(x_{1}, x_{2}, u\right)+\Xi\right| \leq \rho
$$


where $\rho$ is a known positive constant.

Assumption 2. The derivative of the unknown function is bounded by

$$
\left|\dot{\Omega}\left(x_{1}, x_{2}, u, t\right)\right| \leq L
$$

where $L$ is a known positive constant.

The above two assumptions are widely utilized in the design of FTC system in the literature [45], wherein the Lipschitz condition is assumed to be practically satisfied in the bounded region of the state space. In practice, the above two assumptions are somehow true for incipient faults. For abrupts faults, we expect that, due to the high robustness property of the controller, the effects of faults in the system can be mitigated gradually and hence, the above two assumptions can be satisfied. It should be mentioned that if the faults function cannot be bounded as in Assumptions 1 and 2, then it is really challenging to design a fault tolerant controller. These fault conditions are beyond the scope of this paper.

\subsection{Finite-time stability and fixed-time stability and Lemmas}

Consider the following differential equation system:

$$
\dot{x}(t)=f(x(t)), x(0)=x_{0}
$$

where $x \in \Re^{n}$ and $f: \Re^{n} \rightarrow \Re^{n}$ is a nonlinear function. Suppose that the origin is an equilibrium point of 9 .

Definition 1. [43] The equilibrium point of system (9) is said to be a finite time stable equilibrium if the origin is Lyapunov stable and any solution $x(t)$ starting from $x_{0}$ satisfies $\lim _{x \rightarrow \infty} x\left(t, x_{0}\right)=0$ for all $t \geq T\left(x_{0}\right)$, where $T: \Re^{n} \rightarrow \Re^{+}$is called the settling time function.

Definition 2. 43] The equilibrium point of system (9) is said to be a fixed-time stable equilibrium point if it is globally finite time stable and its bounded convergence time $T\left(x_{0}\right)$ satisfies $T\left(x_{0}\right)<T_{\max }$, where $T_{\max }>0$ is a positive number. 
Lemma 1. Consider a scalar differential equation [43] below:

$$
\dot{z}=-k_{1}[z]^{\alpha}-k_{2}[z]^{\gamma}
$$

where $k_{1}>0, k_{2}>0, \alpha>1$ and $0<\gamma<1$. Then, the equation (10) admits a finite time settling time $T_{0}$ uniform with respect to the initial condition $z(0)$ and bounded by

$$
T_{0} \leq T\left(\alpha, \gamma, k_{1}, k_{2}\right):=\frac{1}{k_{1}(\alpha-1)}+\frac{1}{k_{2}(1-\gamma)}
$$

Lemma 2. For $0<\kappa<1$, the following inequality holds [42]

$$
\sum_{1}^{n}\left|x_{i}\right|^{1+\kappa} \geq\left(\sum_{1}^{n}\left|x_{i}\right|^{2}\right)^{\frac{1+\kappa}{2}}
$$

Lemma 3. For $\kappa>1$, the following inequality holds [42]

$$
\sum_{1}^{n}\left|x_{i}\right|^{\kappa} \geq n^{1-\kappa}\left(\sum_{1}^{n}\left|x_{i}\right|\right)^{\kappa}
$$

3. Design of disturbance observer using fixed-time second-order sliding mode observer

\subsection{Fixed-time sliding mode observer for disturbance observer}

Based on the dynamic model (6), a fixed-time sliding mode observer (FxTSMO) can be designed as

$$
\dot{v}=\Lambda u+f\left(x_{1}, x_{2}\right)+(\rho+\varepsilon) \operatorname{sign}(e)+\kappa_{1}[e]^{\gamma_{1}}+\kappa_{2}[e]^{\gamma_{2}}
$$

where $e=x_{2}-v,[e]^{\alpha_{i}}=|e|^{\alpha_{i}} \operatorname{sign}(e), v$ is an estimate of the velocity $x_{2}, \kappa_{i}(i=1,2)>0$ and $0<\gamma_{1}<1$ and $\gamma_{2}>1 . \rho$ is a constant value, which is selected based on Assumption 11 and $\varepsilon$ is a small positive constant. The term $z_{e q}=(\rho+\varepsilon) \operatorname{sign}(e)$ is used to approximate the lumped disturbance $\Omega\left(x_{1}, x_{2}, u, t\right)$.

Theorem 1. Consider the system dynamics described in (6), if the disturbance observer in (14) is used to observe the lumped disturbance component, which includes uncertainties, disturbances and faults, then the stability and convergence of the observer's error is guaranteed and the convergence time is bounded by $T_{0} \leq \frac{1}{2^{\frac{\gamma_{1}+1}{2}} \kappa_{1}\left(\frac{\gamma_{1}+1}{2}-1\right)}+$ $190 \frac{1}{2^{\frac{\gamma_{2}+1}{2}} \kappa_{2}\left(1-\frac{\gamma_{2}+1}{2}\right)}$. 
Proof: From (6) and (14), the estimation error can be computed as

$$
\dot{e}=\Omega\left(x_{1}, x_{2}, u, t\right)-(\rho+\varepsilon) \operatorname{sign}(e)-\kappa_{1}[e]^{\gamma_{1}}-\kappa_{2}[e]^{\gamma_{2}}
$$

Consider a Lyapunov function candidate $V=\frac{1}{2} e^{2}$. The derivative of the Lyapunov function with respect to time can be obtained as

$$
\begin{aligned}
\dot{V} & =e \dot{e}=e\left(\Omega\left(x_{1}, x_{2}, u, t\right)-(\rho+\varepsilon) \operatorname{sign}(e)-\kappa_{1}[e]^{\gamma_{1}}-\kappa_{2}[e]^{\gamma_{2}}\right) \\
& =e \Omega\left(x_{1}, x_{2}, u, t\right)-(\rho+\varepsilon)|e|-\kappa_{1}|e|^{\gamma_{1}+1}-\kappa_{2}|e|^{\gamma_{2}+1} \\
& \leq-2^{\frac{\gamma_{1}+1}{2}} \kappa_{1}\left(\frac{1}{2} e^{2}\right)^{\frac{\gamma_{1}+1}{2}}-2^{\frac{\gamma_{2}+1}{2}} \kappa_{2}\left(\frac{1}{2} e^{2}\right)^{\frac{\gamma_{2}+1}{2}}
\end{aligned}
$$

From (16):

$$
\dot{V}+2^{\frac{\gamma_{1}+1}{2}} \kappa_{1} V^{\frac{\gamma_{1}+1}{2}}+2^{\frac{\gamma_{2}+1}{2}} \kappa_{2} V^{\frac{\gamma_{2}+1}{2}} \leq 0
$$

Therefore, according to Lemma 1, the observer's error $e$ has a finite settling time, and the settling time $T_{0}$ is bounded by

$$
T_{0} \leq \frac{1}{2^{\frac{\gamma_{1}+1}{2}} \kappa_{1}\left(\frac{\gamma_{1}+1}{2}-1\right)}+\frac{1}{2^{\frac{\gamma_{2}+1}{2}} \kappa_{2}\left(1-\frac{\gamma_{2}+1}{2}\right)}
$$

This completes the proof.

When the estimation error $e$ converges to zero, from 15 :

$$
\Omega\left(x_{1}, x_{2}, u, t\right)=(\rho+\varepsilon) \operatorname{sign}(e)=z_{e q}
$$

Therefore, the unknown component $\Omega$, which contains fault function, can be reconstructed based on the equivalent output injection (EOI) $z_{e q}$ in (19). However, the EOI contains the discontinuous term $(\rho+\varepsilon) \operatorname{sign}(e)$, which causes chattering. To eliminate the chattering, a low-pass filter can be employed as

$$
\varphi \dot{z}_{f}(t)+z_{f}(t)=z_{e q}(t)
$$

where $\varphi$ is the filter time constant, and $z_{f}(t)$ is the output of the filter. By adding the results in $\sqrt{14}$ into 20, , the filter's output $z_{f}(t)$ will provide the estimation of the lumped disturbance $\Omega\left(x_{1}, x_{2}, u, t\right)$. 


\subsection{Fixed-time second-order sliding mode observer for disturbance observer}

In the observer (14), the exist of sign function provides chattering when constructing the unknown fault function. Therefore, a low-pass filter is usually required to reconstruct the means of the fault information. To eliminate the needs of the filter in the design, a fixed time second-order sliding mode observer (FxTSOSMO) is introduced in this subsection. The observer is designed based on a super-twisting high-order sliding mode algorithm, as follows:

$$
\dot{v}=\Lambda u+f\left(x_{1}, x_{2}\right)+u_{s t w}+\kappa_{1}[e]^{\gamma_{1}}+\kappa_{2}[e]^{\gamma_{2}}
$$

where $e=x_{2}-v,[e]^{\gamma_{i}}=|e|^{\gamma_{i}} \operatorname{sign}(e), v$ is an estimate of the velocity $x_{2}, \gamma_{1}=\frac{1}{2}, \gamma_{2}>2$, $u_{s t w}$ is used to approximate the unknown component $\Omega$, and it is designed based on a super-twisting algorithm [46]:

$$
\begin{aligned}
& u_{s t w}=\mu_{1}|e|^{\frac{1}{2}} \operatorname{sign}(e)+\xi \\
& \dot{\xi}=k(t) \operatorname{sign}(e)
\end{aligned}
$$

where the gain $\mu_{1}>0$ is selected large enough.

Theorem 2. Consider the system dynamics described in (6), if the disturbance observer in (14) is used to observe the lumped disturbance component, which includes uncertainties, disturbances and faults, then the stability and convergence of the observer's error is guaranteed and the convergence time is bounded by

$$
T_{0} \leq\left(\frac{1}{\kappa_{2}\left(\gamma_{2}-1\right) c^{\gamma-2}}+\frac{2 c^{1 / 2}}{\lambda_{1}}\right) \times\left(1+\frac{1}{m\left((1 / M)-h\left(\lambda_{1}\right) /\left(\lambda_{1}\right)\right)}\right)
$$

where $\lambda_{1}=\kappa_{1}+\mu_{1}, c>0, M=k+L, m=k-L, h\left(\lambda_{1}\right)=1 / \lambda_{1}+\left(2 e_{l} /, \lambda_{1}\right)^{1 / 2}$, and $e_{l}$ is the base natural logarithms, and the control gains satisfied the conditions: $k>L$, $\lambda_{1} h^{-1}\left(\lambda_{1}\right)>M$. The minimum value of $T_{f}(c)$ is reached for $c=\left(\lambda_{1} / \kappa_{2}\right)^{1 /\left(\gamma_{2}+1 / 2\right)}$.

Proof: From (6) and (21), the estimation error can be computed as

$$
\dot{e}=\Omega\left(x_{1}, x_{2}, u, t\right)-u_{s t w}-\kappa_{1}[e]^{\gamma_{1}}-\kappa_{2}[e]^{\gamma_{2}}
$$

The formulation (24) has a form of general system described in equation (5) in the reference [44]. Therefore, the result of Theorem 2 can be proved as a similar way as in [44]. This completes the proof. 
When the estimation error $e$ converges to zero, from (22) and (24), it yields

$$
\begin{aligned}
& \Omega\left(x_{1}, x_{2}, u, t\right)=\xi \\
& \dot{\xi}=k(t) \operatorname{sign}(e)
\end{aligned}
$$

Therefore, the unknown function $\Omega$ can be reconstructed directly from the continuous function $\xi$ without requiring a low-pass filter. Therefore, theoretically, the design of the fault diagnosis observer based on the SOSM observer is generally better than that of the first-order sliding mode observer.

Remark 1. The sliding gain $k(t)$ in $(22)$ was selected based on Assumption 2 However, if the condition in Assumption 2 is not satisfactory (i.e., the bounded value is unknown), the gain can be adapted based on the two-layer adaptive scheme given by [47]:

$$
\begin{aligned}
& \delta(t)=k(t)-\frac{1}{v_{1}}\left|w_{e q}(t)\right|-v_{0}, v_{0}, v_{1}>0 \\
& \dot{k}(t)=-\left(\rho_{0}+\rho(t)\right) \operatorname{sign}(\delta(t)), \rho_{0}>0 \\
& \dot{\rho}=\beta|\delta(t)|, \beta>0
\end{aligned}
$$

where $w_{e q}(t)$ is obtained by low-pass filtering $(L P F)$ of $k(t) \operatorname{sign}(e)$, and $v_{0}, v_{1}, \rho_{0}, \beta>$ 0 are control parameters.

Remark 2. The design of the fixed-time super-twisting second-order sliding mode observer, which can provide a fixed-time convergence of the observer error and provide fault estimation without requiring the use of a low-pass filter, is one of the main contribution of this paper.

Remark 3. The proposed disturbance observer in (21) is used to estimate the lumped disturbance $\Omega\left(x_{1}, x_{2}, u, t\right)$, which includes uncertainties, disturbances and faults. Therefore, when the effects of uncertainties and disturbances are smalll, the proposed disturbance observer tends to approximate the fault function accurately. In this case, the proposed disturbance observer can be considered as a 'fault diagnosis observer'. In practice, a suitable threshold is usually selected to distinguish between the effects of 
the uncertainties, disturbances and faults. This approach has been discussed in our previous work [28].

\section{Design of fault tolerant control based on fixed-time sliding mode control}

\subsection{Fixed-time sliding mode control}

Based on (6) and 21), the dynamic system can be rewritten as

$$
\begin{aligned}
& \dot{x}_{1}=x_{2} \\
& \dot{x}_{2}=\Lambda u+f\left(x_{1}, x_{2}\right)+u_{s t w}+\varepsilon
\end{aligned}
$$

where $\varepsilon=\delta\left(x_{1}, x_{2}, u\right)+\Xi-u_{s t w}$ is the fault estimation error.

Assumption 3. The fault estimation error $\varepsilon$ is bounded by

$$
\varepsilon \leq \Gamma
$$

where $\Gamma$ is a positive constant.

According to the above anaysis, the estimated output of the fault diagnosis observer converges to the real fault function after a fixed time. Therefore, the condition stated in Assumption 3 is satisfactory in the practical applications.

Let $e=x_{1}-x_{d}$ and $\dot{e}=x_{2}-\dot{x}_{d}$, where $x_{d}$ and $\dot{x}_{d}$ are the desired trajectory and the derivative of the desired trajectory, respectively. Then, in order to obtain a fixed-time sliding mode controller, the following sliding surface is selected based on Lemma 1 [48]:

$$
s=\dot{e}+k_{1}[e]^{\alpha}+k_{2}[e]^{\gamma}
$$

where $0<\alpha<1$ and $\gamma>1$. The gains $k_{1}$ and $k_{2}$ are positive constants.

Differentiating (29) with respect to time, we have

$$
\dot{s}=\dot{x}_{2}-\ddot{x}_{d}+k_{1} \alpha|e|^{\alpha-1} \dot{e}+k_{2} \gamma|e|^{\gamma-1} \dot{e}
$$

Inserting the result in 27] into (30), one obtains

$$
\dot{s}=\Lambda u+f\left(x_{1}, x_{2}\right)+u_{s t w}+\varepsilon-\ddot{x}_{d}+k_{1} \alpha|e|^{\alpha-1} \dot{e}+k_{2} \gamma|e|^{\gamma-1} \dot{e}
$$


From (31), the proposed controller is designed as

$$
u=u_{e q}+u_{s}
$$

where, the equivalent controller is designed as

$$
u_{e q}=\Lambda^{-1}\left(-f\left(x_{1}, x_{2}\right)-u_{s t w}+\ddot{x}_{d}-k_{1} \alpha|e|^{\alpha-1} \dot{e}-k_{2} \gamma|e|^{\gamma-1} \dot{e}\right)
$$

And, the switching controller is selected as

$$
u_{s}=\Lambda^{-1}\left(-(\Gamma+a) \operatorname{sign}(s)-\lambda_{1}[s]^{m_{1}}-\lambda_{2}[s]^{m_{2}}\right)
$$

where $\Gamma$ is selected as in Assumption 3 and $a$ is a small positive constant, the constant parameters $\lambda_{1}, \lambda_{2}>0$, the parameters $0<m_{1}<1$ and $m_{2}>1$.

Inserting the composite controller (32), (33) and (34) into (31), yields

$$
\dot{s}=\varepsilon-(\Gamma+a) \operatorname{sign}(s)-\lambda_{1}[s]^{m_{1}}-\lambda_{2}[s]^{m_{2}}
$$

Consider a Lyapunov function candidate below

$$
V=\frac{1}{2} \sum_{1}^{n} s_{i}^{2}
$$

Based on (36), the time derivative of the Lyapunov $V$ can be obtained as

$$
\begin{aligned}
\dot{V} & =\sum_{1}^{n} s_{i} \dot{s}_{i} \\
& \leq \sum_{1}^{n}\left(\varepsilon s_{i}-\Gamma\left|s_{i}\right|-\lambda_{1}\left|s_{i}\right|^{m_{1}+1}-\lambda_{2}\left|s_{i}\right|^{m_{2}+1}\right) \\
& \leq \sum_{1}^{n}\left(-\lambda_{1}\left|s_{i}\right|^{m_{1}+1}-\lambda_{2}\left|s_{i}\right|^{m_{2}+1}\right)
\end{aligned}
$$

Using Lemmas 2 and 3 , we have

$$
\begin{aligned}
& \sum_{1}^{n}\left|s_{i}\right|^{m_{1}+1} \geq n^{\frac{1-m_{1}}{2}}\left(\sum_{1}^{n}\left|s_{i}\right|^{2}\right)^{\frac{1+m_{1}}{2}} \\
& \sum_{1}^{n}\left|s_{i}\right|^{m_{2}+1} \geq\left(\sum_{1}^{n}\left|s_{i}\right|^{2}\right)^{\frac{1+m_{2}}{2}}
\end{aligned}
$$


Inserting the results in (38) and (39) into (37), yields

$$
\dot{V} \leq-\lambda_{1} n^{\frac{1-m_{1}}{2}}\left(V^{\frac{1+m_{1}}{2}}\right)-\lambda_{2}\left(V^{\frac{1+m_{2}}{2}}\right)
$$

From (40, according to Lemma 1, the Lyapunov function in (40) is stable and converged by the time:

$$
T_{r} \leq \frac{1}{\lambda_{1} n^{\frac{1-m_{1}}{2}}\left(\frac{1+m_{1}}{2}-1\right)}+\frac{1}{\lambda_{2}\left(1-\frac{1+m_{2}}{2}\right)}
$$

Then, when the sliding surface, i.e., $s$, converges to zero, from 29]:

$$
\dot{e}=-k_{1}[e]^{\alpha}-k_{2}[e]^{\gamma}
$$

Based on Lemma1 it is provided that the estimation error $e$ converges to zero and the convergence time is bounded by

$$
T_{s} \leq T\left(\alpha, \gamma, k_{1}, k_{2}\right):=\frac{1}{k_{1}(\alpha-1)}+\frac{1}{k_{2}(1-\gamma)}
$$

Theorem 3. Consider the dynamic system described in (6), if the controller is designed as in (32) with the disturbance observer designed in (21), then the system is globally fixed-time stable and the settling time is bounded by

$$
T<T_{\max }=T_{o}+T_{s}+T_{r}
$$

where $T_{o}, T_{S}$ and $T_{r}$ are provided in (18), (41) and (43), respectively.

Proof: It is clear that the result of (44) can be obtained by integrating the results in [17, (42) and 40, altogether. This completes the proof.

4.2. Nonsingular fixed-time sliding mode control

According to 33 , the term $|e|^{\alpha-1} \dot{e}$ provides a singular problem when $e=0$ and $\dot{e} \neq 0$. In order to eliminate the singularity phenomenon, the following sliding surface is proposed:

$$
s=e+\frac{1}{k_{2}^{\gamma}}\left[\dot{e}+k_{1}[e]^{\alpha}\right]^{\frac{1}{\gamma}}
$$

where $k_{1}>0, k_{2}>0, \alpha>1$ and $\frac{1}{2}<\gamma<1$. 
When the sliding surface converges to zero, the following equation is obtained

$$
\dot{e}=-k_{1}[e]^{\alpha}-k_{2}[e]^{\gamma}
$$

and therefore, the sliding surface 45 provides a fixed-time convergence as similar to the sliding surface used in 29].

Differentiating the sliding surface (46) with respect to time, one obtains

$$
\dot{s}=\dot{e}+\frac{1}{k_{2}^{\gamma}}|T(e, \dot{e})|^{\frac{1}{\gamma}-1}\left(\ddot{e}+k_{1}|e|^{\alpha-1} \dot{e}\right)
$$

where $T(e, \dot{e})=\dot{e}+k_{1}[e]^{\alpha}$.

Inserting (27) into (47) yields

$$
\dot{s}=\dot{e}+\frac{1}{k_{2}^{\gamma}}|T(e, \dot{e})|^{\frac{1}{\gamma}-1}\left(\Lambda u+f\left(x_{1}, x_{2}\right)+u_{s t w}+\varepsilon-\ddot{x}_{d}+k_{1}|e|^{\alpha-1} \dot{e}\right)
$$

The proposed controller is designed as

$$
u=u_{0}+u_{c}+u_{s}
$$

where,

$$
u_{0}=\Lambda^{+}\left(-f\left(x_{1}, x_{2}\right)-u_{s t w}+\ddot{x}_{d}-k_{1}|e|^{\alpha-1} \dot{e}\right)
$$

where $\Lambda^{+}$is the pseudo inverse of the $\Lambda$, and

$$
u_{c}=-\Lambda^{+} k_{2}^{\gamma}|T(e, \dot{e})|^{1-\frac{1}{\gamma}} \dot{e}
$$

and,

$$
u_{s}=\Lambda^{+}\left(-(\Gamma+a) \operatorname{sign}(s)-\lambda_{1}[s]^{m_{1}}-\lambda_{2}[s]^{m_{2}}\right)
$$

where $a$ is a small positive constant.

Inserting the controller (49) into (48), yields

$$
\begin{aligned}
\dot{s} & =\frac{1}{k_{2}^{\gamma}}|T(e, \dot{e})|^{\frac{1}{\gamma}-1}\left(\varepsilon-(\Gamma+a) \operatorname{sign}(s)-\lambda_{1}[s]^{m_{1}}-\lambda_{2}[s]^{m_{2}}\right) \\
& =g\left(\varepsilon-(\Gamma+a) \operatorname{sign}(s)-\lambda_{1}[s]^{m_{1}}-\lambda_{2}[s]^{m_{2}}\right)
\end{aligned}
$$

where $g=\frac{1}{k_{2}^{\gamma} \mid}|T(e, \dot{e})|^{\frac{1}{\gamma}-1}$.

In 51 , there is a negative fractional power term $|T(e, \dot{e})|^{1-\frac{1}{\gamma}} \dot{e}$ exists, however this term does not provide singularity phenomenon. Actually, when $\dot{e}=0, e \neq 0$, we have $|\dot{e}|^{1-\frac{1}{\gamma}} \dot{e} \geq \dot{e}^{2-\frac{1}{\gamma}}, 2-\frac{1}{\gamma}$ is a positive power term. 
Theorem 4. Consider the system (6) and the proposed sliding surface (45). The system states of the closed-loop system, which includes the proposed observer (21) and the proposed controller (49), are stable and convergent to the origin within a fixed time and the settling time $T$ is bounded by

$$
T<T_{\max }=T_{o}+T_{r}+T_{s}+\varepsilon(\tau)
$$

245 to the boundary width $\tau=\left(k_{2}^{\gamma}\right)^{\frac{\gamma}{\gamma-1}}$.

Proof: Consider a Lyapunov function candidate

$$
V=\frac{1}{2} \sum_{1}^{n} s_{i}^{2}
$$

Differentiating the Lyapunov function (55) with respect to time and combining with (53), yields

$$
\begin{aligned}
\dot{V} & =\sum_{1}^{n} s_{i} \dot{s}_{i} \\
& \leq g \sum_{1}^{n}\left(\varepsilon s_{i}-\Gamma\left|s_{i}\right|-\lambda_{1}\left|s_{i}\right|^{m_{1}+1}-\lambda_{2}\left|s_{i}\right|^{m_{2}+1}\right) \\
& \leq g \sum_{1}^{n}\left(-\lambda_{1}\left|s_{i}\right|^{m_{1}+1}-\lambda_{2}\left|s_{i}\right|^{m_{2}+1}\right) \\
& \leq g\left(-\lambda_{1} n^{\frac{1-m_{1}}{2}}\left(V^{\frac{1+m_{1}}{2}}\right)-\lambda_{2}\left(V^{\frac{1+m_{2}}{2}}\right)\right)
\end{aligned}
$$

Next, we will show that 56 is fixed time stable. The idea of this proof is inspired from [42].

For the case of $T(e, \dot{e}) \neq 0$, i.e, $g>0$. For ease of the proof, the working state space $250 \quad(e, \dot{e})$ is divided into two different areas $S_{1}=(e, \dot{e}) \mid g \geq 1$ and $S_{2}=(e, \dot{e}) \mid g<1$.

(i) When the system states are in the region of $S_{1}$, we have

$$
\dot{V} \leq-\lambda_{1} n^{\frac{1-m_{1}}{2}}\left(V^{\frac{1+m_{1}}{2}}\right)-\lambda_{2}\left(V^{\frac{1+m_{2}}{2}}\right)
$$

This implies that the system is fixed time stable and the convergence time is bounded as in 41.

(ii) When the system states are in the region of $S_{2}$ when $T(e, \dot{e}) \neq 0$, according to (56), the terminal sliding surface $s=0$ is still an attractor. What remains is to prove 


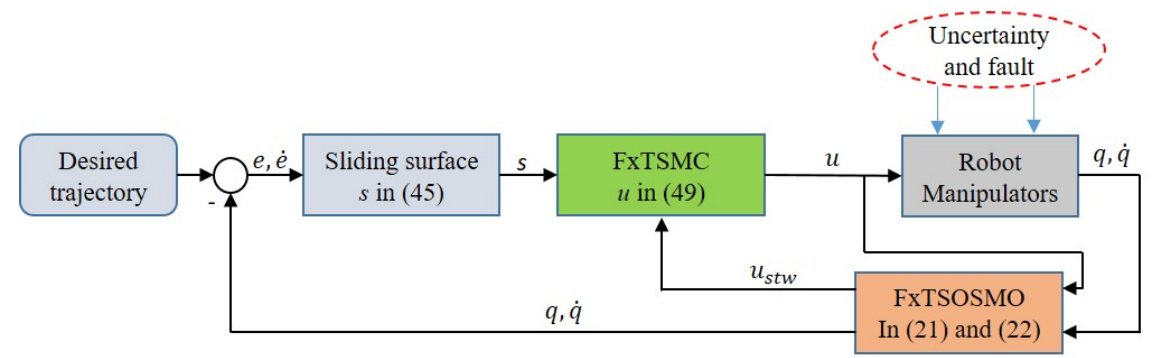

Figure (1) The block diagram of the proposed closed-loop FxTSOSMO-FxTSMC paper. That is the convergence of the closed-loop fault diagnosis-fault tolerant control system can be convergent after a fixed-time, which is unprecedented.

Remark 5. In the controller in (52), the presence of the sign function provide chattering in the system. In order to reduce the chattering, the boundary method can be employed. Thus, the controller in (52) can be modified as

$$
u_{s}=\Lambda^{+}\left(-(\Gamma+a) \frac{s}{|s|+c_{s}}-\lambda_{1}[s]^{m_{1}}-\lambda_{2}[s]^{m_{2}}\right)
$$

where $c_{s}$ is a small positive constant.

Remark 6. From (24) and (25), the observer's error of the proposed observer (21) 270 will converge to zero no matter what the control input is. It means that the design 
of the observer does not depend on the control input. Therefore, the stability of the closed-loop observer-controller in Theorem 4 can be guaranteed as in the proof.

Remark 7. In summary, in terms of tool, three novelties has been reported in this paper compared to recent works in this domain. First, a fixed-time second-order sliding mode observer is proposed for first time. This proposed technique is significant since it can provide faster convergence speed of the observer's error. In addition, the bounded value of the convergence time can be predicted in advance. Second, a fixed-time sliding mode control is proposed. Actually, the theory of the fixed-time sliding mode control is not new, but its applications for FTC of robot manipulators is really new. Third, this is the first method to provide a global fixed time convergence of the closed-loop observer-controller for FTC systems.

\section{Simulation and Results}

In order to verify the performance of the system, we employ the proposed method for fault diagnosis and fault tolerant control system of a PUMA560 robot. The PUMA560 robot is a well-known robot and it has been widely used as a benchmark platform for research and development. The PUMA560 robot has six joints but for shortening the presentation of the simulation results, only the first three joints are used in this study. The kinematic and dynamic model of the robot are taken from [49]. The dynamic model of the robot can be described as in 11 with the nominal parameters are taken from [49]. To verify and compare the performance of the disturbance observer to approximate an unknown fault function and the effectiveness of the proposed fault tolerant controller, the friction term of the robot and external disturbance are neglected first in this simulation section. Hence, the unknown component $\Omega$ contains the faults' effects only. In order to illustrate the faults' effects in the system, the following fault function is selected:

$$
\phi_{1}(q, \dot{q}, \tau)=\left[\begin{array}{cc}
{[9 \sin (0.5 t)+8.5 \cos (0.5 t)+12 \cos (0.5 t)],} & t \geq 10 \\
-0.5 \tau_{2}, & t \geq 20 \\
-0.35 \tau_{3}, & t \geq 20
\end{array}\right]
$$


The desired trajectory of the robot is defined in advance as

$$
x_{d}=\left[\cos (t / 5 \pi)-1, \cos \left(t / 5 \pi+\frac{\pi}{2}\right), \sin \left(t / 5 \pi+\frac{\pi}{2}\right)-1\right]^{T}
$$

In this section, the two major innovative schemes in this paper will be verified: the proposed disturbance observer acted as a fault diagnosis observer, as discussed in Remark 3 , and the proposed fault tolerant control. First, the effectiveness of the proposed fault diagnosis observer is discussed. In this paper, we compare the performances of the FxTSMO and the FxTSOSMO. Since the performances of the observers are dependent on the selection of the sliding gain, to be fair in comparison, we use the adaptive law described in 26, to tune the gain $\rho$ in 14 of the FxTSMO $(\rho=k(t))$ and the gain $k(t)$ in 22 of the FxTSOSMO. The parameters of the two observers are selected as follows. For the FxTSMO: $\kappa_{1}=20, \kappa_{2}=20, \gamma_{1}=0.75, \gamma_{2}=1.5$, and for the FxTSOSMO: $\kappa_{1}=10, \kappa_{2}=20, \mu_{1}=10, \gamma_{1}=0.5, \gamma_{2}=2.5$. The parameters of the adaptive gains of the fault diagnosis observer in 26 are selected as $v_{0}=3, v_{1}=1.9, \rho_{0}=3, \beta=5$. Under the selections of the parameters, the fault estimation performances of the two observers, i.e., FXTSMO and FxTSOSMO, are shown in Fig. 2. As the results shown in Fig. 2, it can be seen that the use of low-pass filter reduces the estimation accuracy of the FxTSMO significantly. In contrast, the proposed FxTSOSMO provides very high accuracy of fault estimation. Therefore, it can be concluded that the FxTSOSMO provides better fault estimation accuracy than that of the FxTSMO. The time history of the adaptive gain $k(t)$ is shown in Fig. 3 . From Fig. 3 it can be observed that the adaptive gains adapt to the variations of the fault functions very well.

Next, the tracking performance of the proposed global fixed time sliding mode control (GFxTSMC) is verified. To easily analyse the outstanding properties of the GxFTSMC, we compare it with two popular fault tolerant control schemes: computed torque control (CTC) and nonsingular fast terminal sliding mode control (NFTSMC). The designs of the CTC and the NFTSMC can be referred to [40]. The parameters of the controllers are selected as follows. For the CTC, the gains are selected as $K_{p}=$ $200, K_{d}=10$, and for the NFTSMC, the parameters are selected as $k_{1}=10, k_{2}=5, \lambda=$ $1.4, p=9, q=7$ [40]. The parameters of the proposed GFXTSMC are selected as $k_{1}=3, k_{2}=3, \alpha=1.75, \gamma=0.75$, and the gains $\Gamma=50, a=1$. To reduce the chattering, 
the gain $c_{s}$ is selected as $c_{s}=0.05$.

The tracking performance of the three controllers, i.e., CTC, NFTSMC and GFxTSMC, in cartesian space is illustrated in Fig. 4. For sake of comparison, the tracking errors of the three controllers are also reported in Fig. 5 . From Figs. 4 and 5 , it can be seen that, when the system in normal operation, the CTC provides an acceptable performance. However, when the fault occurs, the CTC fails to maintain the desired performance. In contrast, the NFTSMC provides better fault accommodation capability than that of the CTC. This is due to the high robustness property of the SMC compared to the CTC. However, it does not provide high performance for some instances; for example, the poor tracking response in the first joint shown in Fig. 5. It means the NFTSMC does not possess high robustness against a fault function with high magnitude. By using the fault information obtained from the fault diagnosis observer, the proposed GFxTSMC provides very good tracking precision in both normal and fault operations. As shown in Figs. 4 and 5 , the effects of faults are compensated very quickly. Therefore, it can be concluded that, in this simulation, the robustness of the proposed FxTSMC is higher compared to the CTC and NFTSMC.

The control inputs of the CTC, NFTSMC and GFxTSMC are shown in Figs. 6, 7 and 8 , respectively. It can be observed that, while the CTC provides smooth control input because it does not contain the 'sign' function in the design, the NFTSMC and the GFxTSMC also provide smooth control efforts by using the boundary method designed in (58). Therefore, from the results shown in the above figures, it can be concluded that the proposed GFxTSMC provides outstanding fault tolerant capability and smooth control efforts.

To futher show the effectiveness of the proposed method, we consider the effects of the lumped disturbance, which includes the friction component $F(q, \dot{q})$, the external disturbance $\tau_{d}$ and the fault function $\phi_{2}(q, \dot{q}, \tau)$ :

$$
F(q, \dot{q})=\left[\begin{array}{c}
1.5 \dot{q}_{1}+\sin \left(3 q_{1}\right)+1.5 \sin \left(\dot{q}_{1}\right) \\
1.5 \dot{q}_{2}-2 \sin \left(2 q_{1}\right)+1.3 \sin \left(\dot{q}_{2}\right) \\
-1.2 \dot{q}_{3}-2 \sin \left(q_{3}\right)+1.13 \sin \left(\dot{q}_{3}\right)
\end{array}\right]
$$


and the external disturbance component is assumed:

$$
\tau_{d}=\left[\begin{array}{c}
2.4 \cos (t)+1.5 \sin (t) \cos (t) \\
1.5 \cos ^{2}(t)+3 \cos (t) \\
2.5 \sin (t)-2.2 \sin (t) \cos (t)
\end{array}\right]
$$

and, a new fault function is instroduced in the system:

$$
\phi_{2}(q, \dot{q}, \tau)=\left[\begin{array}{ll}
-0.7 \tau_{1}, & t \geq 10 \\
-0.7 \tau_{2}, & t \geq 20 \\
-0.7 \tau_{3}, & t \geq 20
\end{array}\right]
$$

Because the NFTSMC provided better performance than the CTC as shown in the previous experiment, in this working condition, the performances of the NFTSMC and the proposed GFTSMC are compared. The tracking performance and the tracking error of the system under the inputs of the NFTSMC and the proposed GFTSMC are shown in Figs. 9 and 10 respectively. It can be seen from Figs. 9 and 10 that the NFTSMC provides worst tracking performance for this severe condition. However, the proposed GFTSMC provides very good tracking performance. It demonstrates the high robustness property of the proposed approach. The control inputs of the controllers are omitted in this simulation to reduce the length of the paper.

\section{Conclusions}

A robust fault tolerant control with global fixed-time convergence has been proposed for robot manipulators for the first time. The proposed method is constructed based on an integration between a fixed-time second-order sliding mode observer and a fixed-time sliding mode controller. The global fixed-time convergence is guaranteed and can be computed in advance. The stability of the closed-loop observer-controller is proved rigorously. The simulation results on a PUMA560 robot and the comprehensive comparison results with other state-of-the-art observers and controllers verified that the proposed method estimated and tackled the effects of the faults very well.

In this paper, the constraints of the control inputs and outputs of the system have not been considered yet. In addition, the effects of other faults such as sensor faults 


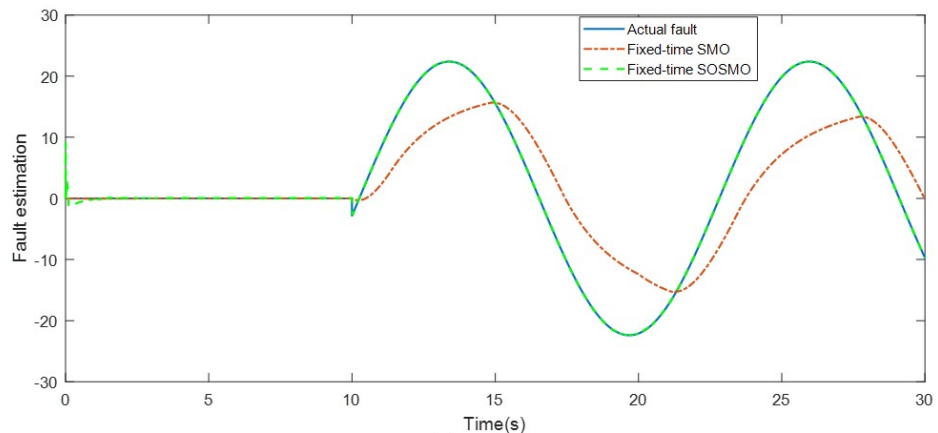

(a) Joint 1

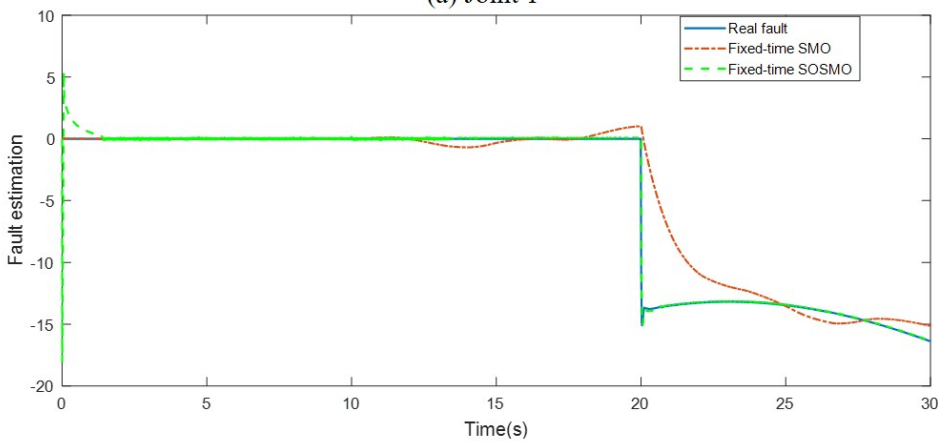

(b) Joint 2

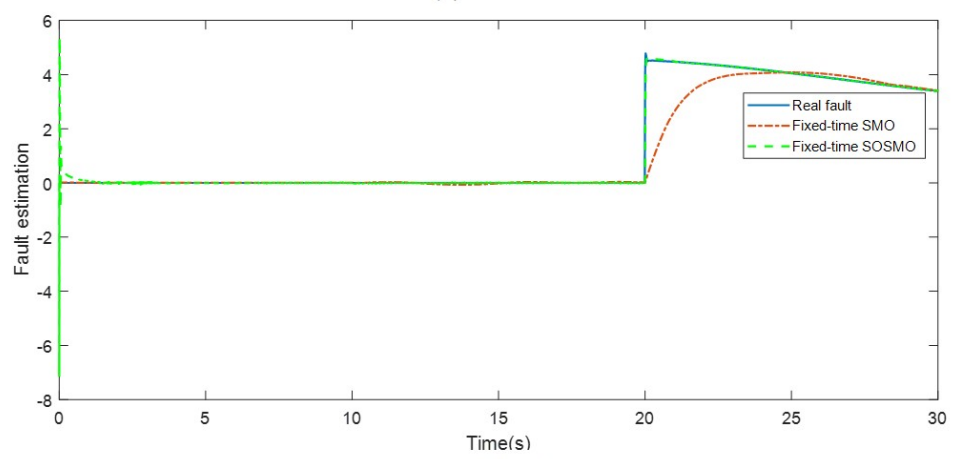

(c) Joint 3

Figure (2) Fault estimations using the fixed-time SMO and fixed-time SOSMO

were neglected when designing the proposed observer and controller. These issues will be further studied in our future works.

\section{Conflict of interest}

The authors declare that they have no conflict of interest. 


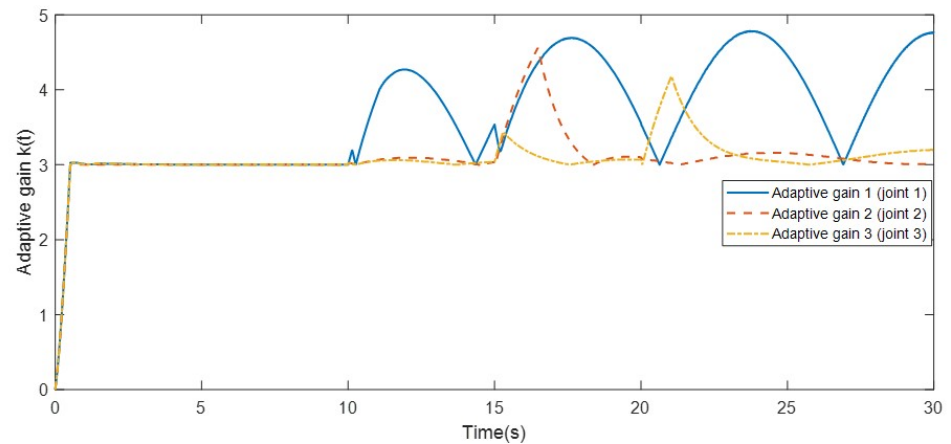

Figure (3) Time history of the adaptive gain $k(t)$ of the fixed-time SOSMO
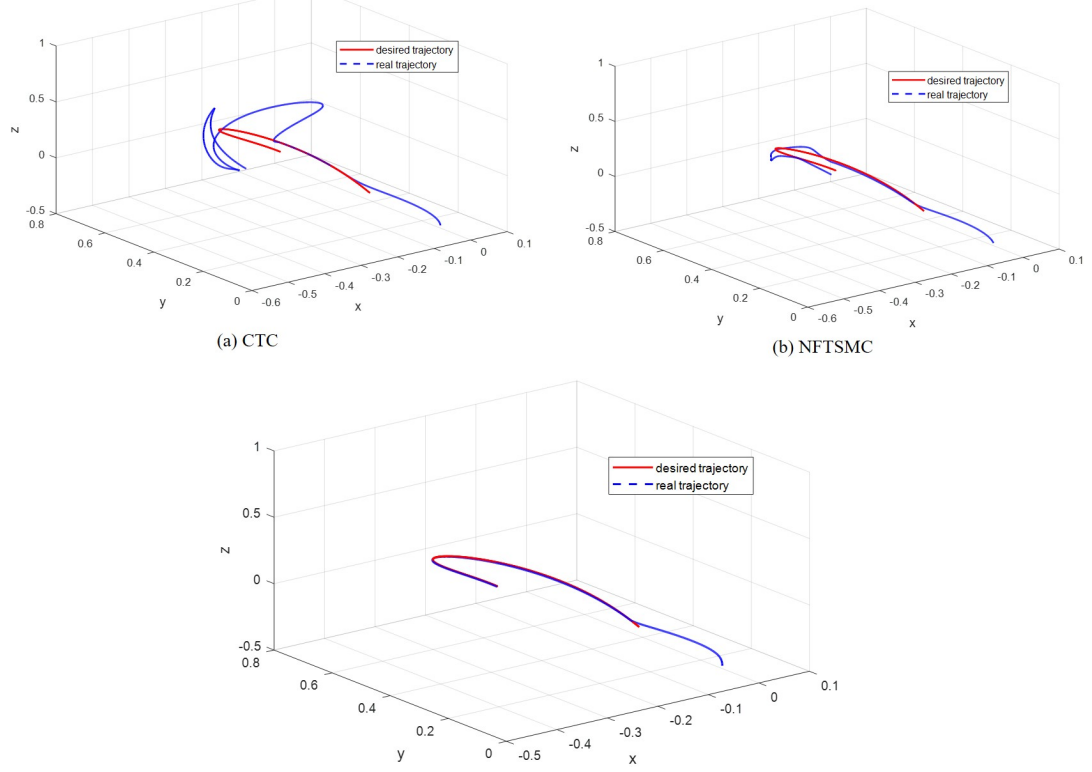

(c) Proposed GFTSMC

Figure (4) Tracking performances of the controllers under the effects of the fault

\section{References}

[1] C. Tawk, G. Spinks, M. in het Panhuis, G. Alici, 3d printable linear soft vacuum actuators (lsova): their modeling, performance quantification and application in soft robotic systems, IEEE/ASME Transactions on Mechatronics (2019) 1-1doi: 10.1109/TMECH. 2019.2933027 


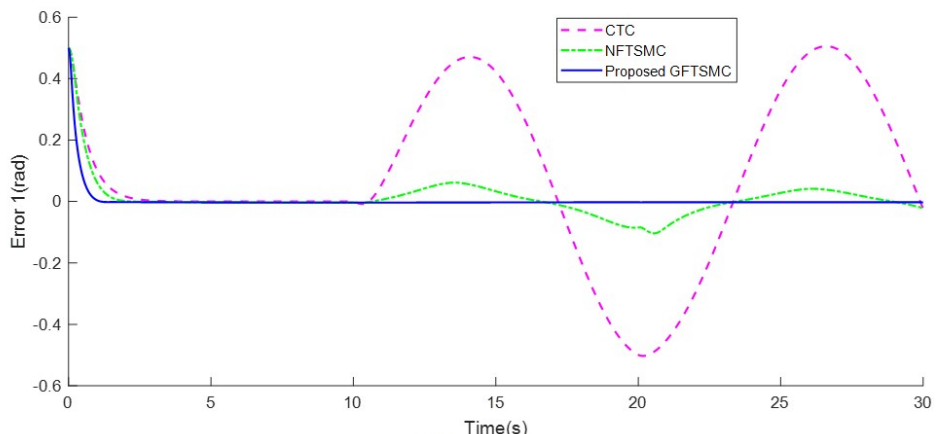

(a) Joint 1

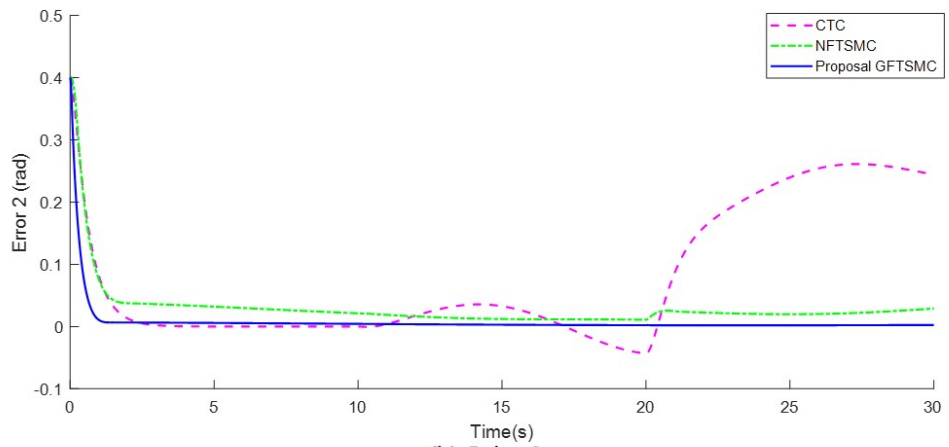

(b) Joint 2

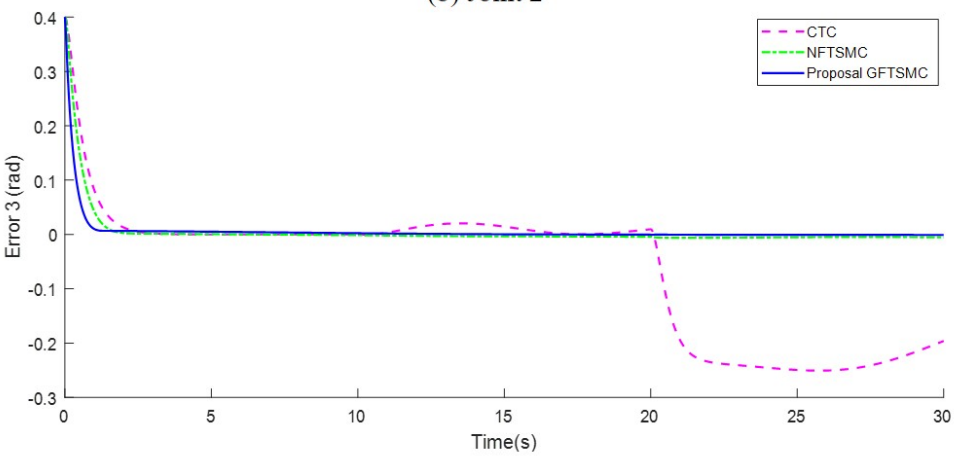

(c) Joint 3

Figure (5) Comparison in tracking errors among the controllers under the effects of the fault

[2] L. Biagiotti, L. Moriello, C. Melchiorri, Improving the accuracy of industrial robots via iterative reference trajectory modification, IEEE Transactions on Control Systems Technology (2019) 1-13doi:10.1109/TCST .2019.2892929.

[3] F. Müller, J. Jakel, J. Suchy, U. Thomas, Stability of nonlinear time-delay systems describing human-robot interaction, IEEE/ASME Transactions on Mechatronics 


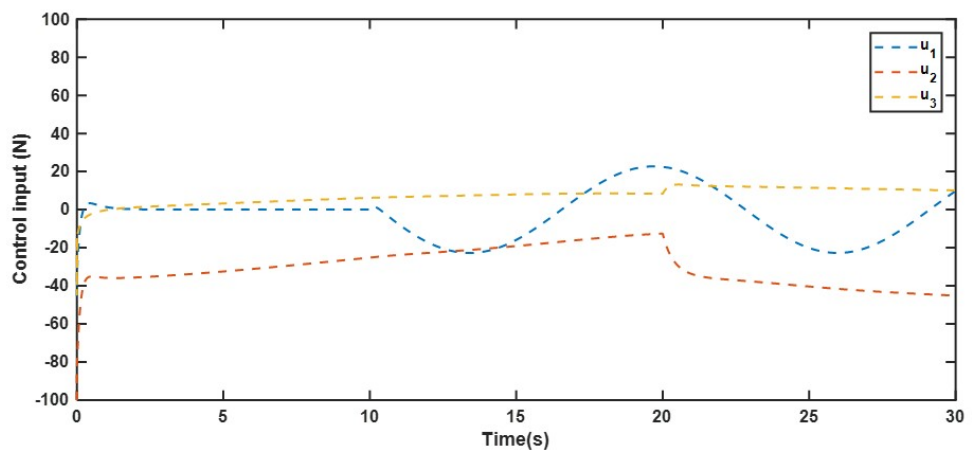

Figure (6) Control input of the Computed Torque Controller

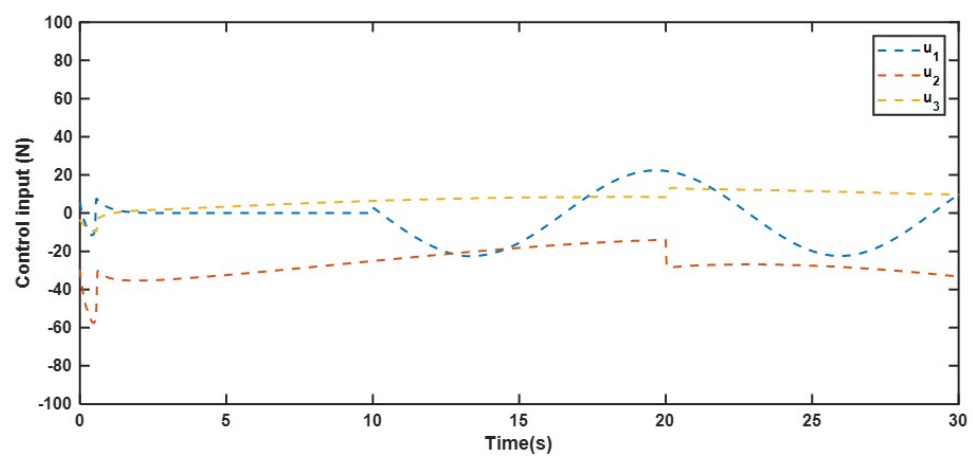

Figure (7) Control input of the NFTSMC Controller

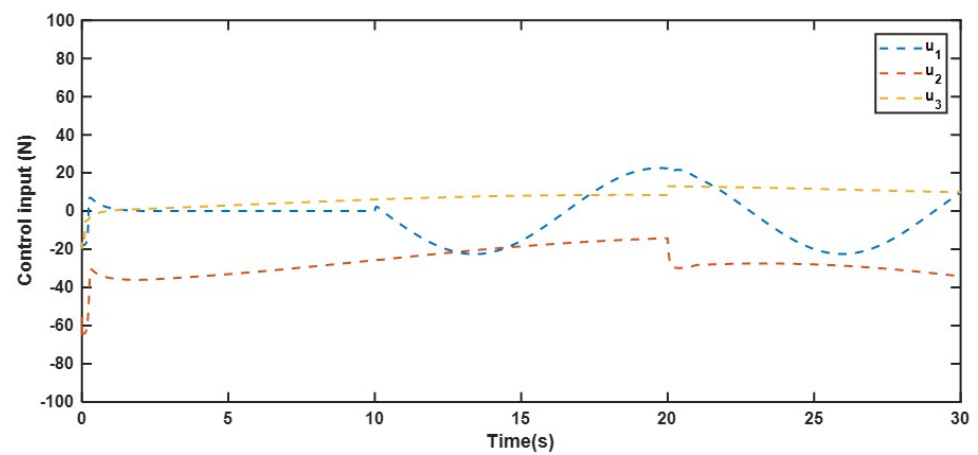

Figure (8) Control input of the NFTSMC Controller

(2019) 1-1doi : 10.1109/TMECH. 2019.2939907.

[4] L. Han, W. Xu, B. Li, P. Kang, Collision detection and coordinated compli- 


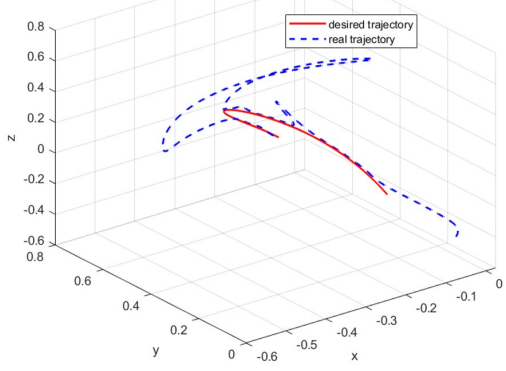

(a) NFTSMC

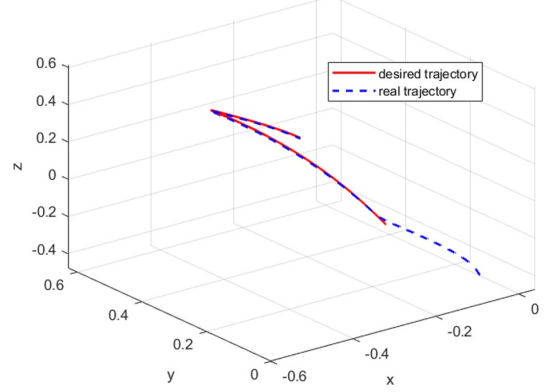

(b) Proposed GFTSMC

Figure (9) Tracking performances of the NFTSMC and proposed GFTSMC controllers under the effects of the lumped disturbance

[9] D. Ceglarek, M. Colledani, J. Váncza, D.-Y. Kim, C. Marine, M. Kogel-

ance control for dual-arm robot without force/torque sensing based on momentum observer, IEEE/ASME Transactions on Mechatronics (2019) 1-1doi: 10.1109/TMECH. 2019.2934141

[5] S. Lee, Y. Yamada, K. Ichikawa, O. Matsumoto, K. Homma, E. Ono, Safetyfunction design for the control system of a human-cooperative robot based on functional safety of hardware and software, IEEE/ASME Transactions on Mechatronics 19 (2) (2014) 719-729.

[6] L. M. Capisani, A. Ferrara, A. F. de Loza, L. M. Fridman, Manipulator fault diagnosis via higher order sliding-mode observers, IEEE Transactions on Industrial Electronics 59 (10) (2012) 3979-3986.

[7] F. Caccavale, A. Marino, G. Muscio, F. Pierri, Discrete-time framework for fault diagnosis in robotic manipulators, IEEE Transactions on Control Systems Technology 21 (5) (2013) 1858-1873.

[8] M. Van, D. Wu, S. S. Ge, H. Ren, Fault diagnosis in image-based visual servoing with eye-in-hand configurations using kalman filter, IEEE Transactions on Industrial Informatics 12 (6) (2016) 1998-2007. 


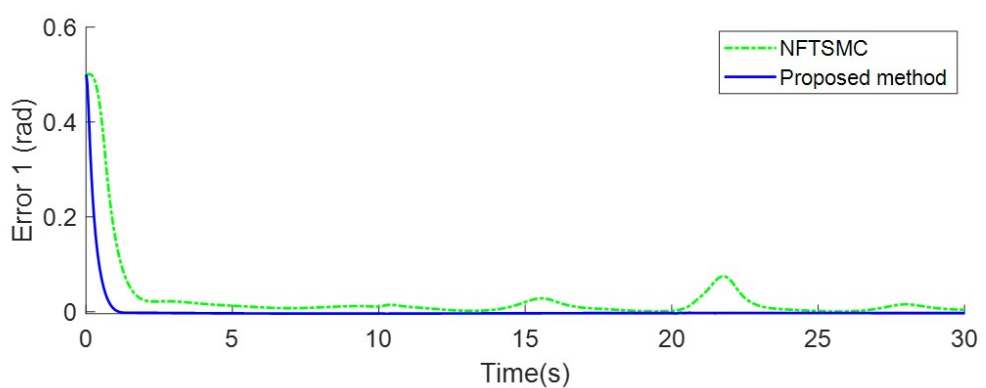

(a) Joint 1
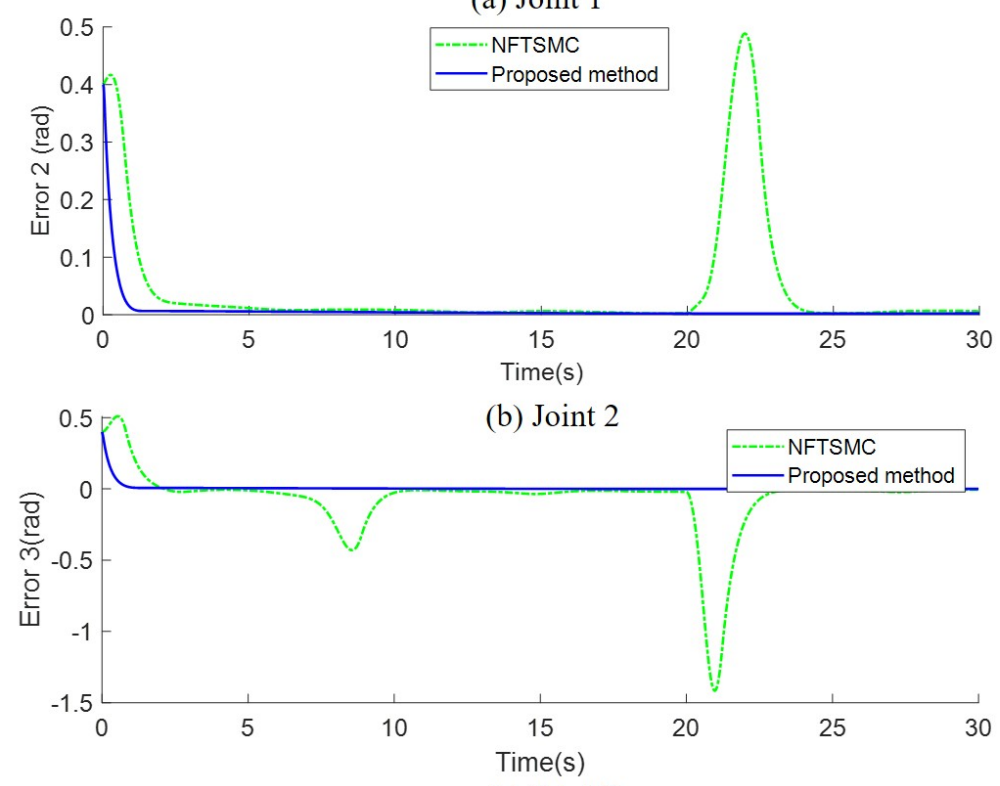

(c) Joint 3

Figure (10) Tracking performances of the NFTSMC and proposed GFTSMC controllers under the effects of the lumped disturbance

Hollacher, A. Mistry, L. Bolognese, Rapid deployment of remote laser welding processes in automotive assembly systems, CIRP Annals 64 (1) (2015) 389 - 394.

[10] P. Franciosa, A. Serino, R. A. Botros, D. Ceglarek, Closed-loop gap bridging control for remote laser welding of aluminum components based on first principle energy and mass balance, Journal of Laser Applications 31 (2) (2019) 022416. arXiv:https://doi.org/10.2351/1.5096099

[11] Yu Ding, Pansoo Kim, D. Ceglarek, Jionghua Jin, Optimal sensor distribution 
for variation diagnosis in multistation assembly processes, IEEE Transactions on Robotics and Automation 19 (4) (2003) 543-556.

[12] M. Babu, P. Franciosa, D. Ceglarek, Spatio-temporal adaptive sampling for effective coverage measurement planning during quality inspection of free form surfaces using robotic 3d optical scanner, Journal of Manufacturing Systems 53 (2019) $93-108$.

[13] E. Glorieux, P. Franciosa, D. Ceglarek, Coverage path planning with targetted viewpoint sampling for robotic free-form surface inspection, Robotics and Computer-Integrated Manufacturing 61 (2020) 101843.

[14] E. Glorieux, P. Franciosa, D. Ceglarek, Quality and productivity driven trajectory optimisation for robotic handling of compliant sheet metal parts in multi-press stamping lines, Robotics and Computer-Integrated Manufacturing 56 (2019) 264 -275 .

[15] Z. Gao, C. Cecati, S. X. Ding, A survey of fault diagnosis and fault-tolerant techniques, part ii: Fault diagnosis with knowledge-based and hybrid/active approaches, IEEE Transactions on Industrial Electronics 62 (6) (2015) 3768-3774.

[16] M. Van, An enhanced robust fault tolerant control based on an adaptive fuzzy pidnonsingular fast terminal sliding mode control for uncertain nonlinear systems,

a IEEE/ASME Transactions on Mechatronics 23 (3) (2018) 1362-1371. doi:10. 1109/TMECH. 2018.2812244.

[17] H. Yang, B. Jiang, H. H. T. Liu, H. Yang, Q. Zhang, Attitude synchronization for multiple 3-dof helicopters with actuator faults, IEEE/ASME Transactions on Mechatronics 24 (2) (2019) 597-608. doi:10.1109/TMECH. 2019.2895222

[18] M. Van, S. S. Ge, D. Ceglarek, Fault estimation and accommodation for virtual sensor bias fault in image-based visual servoing using particle filter, IEEE Transactions on Industrial Informatics 14 (4) (2018) 1312-1322. 
[21] H. Ma, Y. Liu, T. Li, G. Yang, Nonlinear high-gain observer-based diagnosis and compensation for actuator and sensor faults in a quadrotor unmanned aerial vehicle, IEEE Transactions on Industrial Informatics 15 (1) (2019) 550-562.

[22] H. A. Talebi, K. Khorasani, S. Tafazoli, A recurrent neural-network-based sensor

[24] S. Huang, D. Zhang, L. Guo, L. Wu, Convergent estimation mechanism design for nonlinear fuzzy systems with faults, IEEE Transactions on Cybernetics (2018) 1-10doi:10.1109/TCYB.2018.2884221.

[25] S. K. Kommuri, S. B. Lee, K. C. Veluvolu, Robust sensors-fault-tolerance with

19] M. Van, H.-J. Kang, Y.-S. Suh, K.-S. Shin, A robust fault diagnosis and accommodation scheme for robot manipulators, International Journal of Control, Automation and Systems 11 (2) (2013) 377-388.

[20] A. Bregon, C. J. Alonso-González, B. Pulido, Integration of simulation and state observers for online fault detection of nonlinear continuous systems, IEEE Transand actuator fault detection and isolation for nonlinear systems with application to the satellite's attitude control subsystem, IEEE Transactions on Neural Networks 20 (1) (2009) 45-60.

[23] S. Huang, D. Zhang, L. Guo, L. Wu, Convergent fault estimation for linear systems with faults and disturbances, IEEE Transactions on Automatic Control 63 (3) (2018) 888-893. doi:10.1109/TAC. 2017.2735547.

sliding mode estimation and control for pmsm drives, IEEE/ASME Transactions on Mechatronics 23 (1) (2018) 17-28.

[26] M. Van, H.-J. Kang, Y.-S. Suh, A novel neural second-order sliding mode observer for robust fault diagnosis in robot manipulators, International Journal of Precision Engineering and Manufacturing 14 (3) (2013) 397-406.

45 [27] C. Lin, C. Chen, Robust fault-tolerant control for a biped robot using a recurrent cerebellar model articulation controller, IEEE Transactions on Systems, Man, and Cybernetics, Part B (Cybernetics) 37 (1) (2007) 110-123. 
[28] M. Van, H.-J. Kang, Y.-S. Suh, K.-S. Shin, A robust fault diagnosis and accommodation scheme for robot manipulators, International Journal of Control, Automation and Systems 11 (2) (2013) 377-388.

[29] H. Gao, Y. Song, C. Wen, Backstepping design of adaptive neural fault-tolerant control for mimo nonlinear systems, IEEE Transactions on Neural Networks and Learning Systems 28 (11) (2017) 2605-2613.

[30] and, Robust adaptive control of robot manipulators using generalized fuzzy neural networks, IEEE Transactions on Industrial Electronics 50 (3) (2003) 620-628.

[31] X. Wang, C. P. Tan, F. Wu, J. Wang, Fault-tolerant attitude control for rigid spacecraft without angular velocity measurements, IEEE Transactions on Cybernetics (2019) 1-14doi:10.1109/TCYB.2019.2905427

[32] H. Badihi, S. Jadidi, Y. Zhang, P. Pillay, S. Rakheja, Fault-tolerant cooperative control in a wind farm using adaptive control reconfiguration and control real-

【 location, IEEE Transactions on Sustainable Energy (2019) 1-1doi:10.1109/ TSTE.2019.2950681,

[33] J. Qin, Q. Ma, H. Gao, W. X. Zheng, Fault-tolerant cooperative tracking control via integral sliding mode control technique, IEEE/ASME Transactions on Mechatronics 23 (1) (2018) 342-351. doi : 10.1109/TMECH. 2017 .2775447

[34] S. K. Kommuri, S. B. Lee, K. C. Veluvolu, Robust sensors-fault-tolerance with sliding mode estimation and control for pmsm drives, IEEE/ASME Transactions on Mechatronics 23 (1) (2018) 17-28. doi:10.1109/TMECH. 2017.2783888.

[35] C. Huang, F. Naghdy, H. Du, Fault tolerant sliding mode predictive control for uncertain steer-by-wire system, IEEE Transactions on Cybernetics (2017) 112doi:10.1109/TCYB.2017.2771497

[36] Q. Meng, T. Zhang, X. Gao, J. y. Song, Adaptive sliding mode fault-tolerant control of the uncertain stewart platform based on offline multibody dynamics, IEEE/ASME Transactions on Mechatronics 19 (3) (2014) 882-894. 
[37] M. Van, S. S. Ge, Adaptive fuzzy integral sliding mode control for robust fault tolerant control of robot manipulators with disturbance observer, IEEE Transactions on Fuzzy Systems (2020) 1-1doi:10.1109/TFUZZ.2020 . 2973955

[38] H. Hou, X. Yu, L. Xu, R. Chuei, Z. Cao, Discrete-time terminal sliding-mode tracking control with alleviated chattering, IEEE/ASME Transactions on Mechatronics 24 (4) (2019) 1808-1817. doi:10.1109/TMECH.2019.2928642

[39] N. Wang, H. R. Karimi, H. Li, S. Su, Accurate trajectory tracking of disturbed surface vehicles: A finite-time control approach, IEEE/ASME Transactions on Mechatronics 24 (3) (2019) 1064-1074. doi:10.1109/TMECH. 2019.2906395

[40] M. Van, M. Mavrovouniotis, S. S. Ge, An adaptive backstepping nonsingular fast terminal sliding mode control for robust fault tolerant control of robot manipulators, IEEE Transactions on Systems, Man, and Cybernetics: Systems (2018) $1-11$.

[41] M. Van, S. S. Ge, H. Ren, Finite time fault tolerant control for robot manipulators using time delay estimation and continuous nonsingular fast terminal sliding mode control, IEEE Transactions on Cybernetics 47 (7) (2017) 1681-1693.

[42] H. Li, Y. Cai, On sftsm control with fixed-time convergence, IET Control Theory Applications 11 (6) (2017) 766-773.

[43] Z. Zuo, Non-singular fixed-time terminal sliding mode control of non-linear systems, IET Control Theory Applications 9 (4) (2015) 545-552.

[44] M. Basin, C. Bharath Panathula, Y. Shtessel, Multivariable continuous fixed-time second-order sliding mode control: design and convergence time estimation, IET Control Theory Applications 11 (8) (2017) 1104-1111.

[45] B. Jiang, M. Staroswiecki, V. Cocquempot, Fault accommodation for nonlinear dynamic systems, IEEE Transactions on Automatic Control 51 (9) (2006) 15781583. 
[46] M. V. Basin, P. Yu, Y. B. Shtessel, Hypersonic missile adaptive sliding mode control using finite- and fixed-time observers, IEEE Transactions on Industrial Electronics 65 (1) (2018) 930-941.

[47] C. Edwards, Y. Shtessel, Adaptive dual-layer super-twisting control and observation, International Journal of Control 89 (9) (2016) 1759-1766.

[48] Z. Zuo, Nonsingular fixed-time consensus tracking for second-order multi-agent networks, Automatica 54 (2015) $305-309$.

[49] B. Armstrong, O. Khatib, J. Burdick, The explicit dynamic model and inertial parameters of the puma 560 arm, in: Proc. IEEE Int. Conf. Robot. Autom, Vol. 3, 1986, pp. 519-518. 